\title{
ANALYTIC FUNCTIONS CLOSE TO MAPPINGS CONVEX IN ONE DIRECTION
}

\author{
WALTER HENGARTNER AND GLENN SCHOBER ${ }^{1}$
}

ABSTRACT. By analogy to the class of close-to-convex functions we define a class of analytic functions which are close to a family $\Sigma$ of mappings onto domains convex in one direction. In contrast to the close-to-convex class the close-to- $\Sigma$ functions are not necessarily univalent. However, we determine the radius of convexity for $\Sigma$, and this gives a lower bound for the radius of univalence of close-to- $\Sigma$ functions. We next derive the coefficient estimate $\left|A_{n}\right| \leqq n\left|A_{1}\right|$ for close-to- $\Sigma$ functions and conclude with an elementary distortion theorem.

1. Mappings convex in one direction. Let $\Sigma$ be the family of nonconstant analytic functions $f$ on the unit disk $U=\{|z|<1\}$ satisfying the condition

$$
\operatorname{Re}\left\{\left(1-z^{2}\right) f^{\prime}(z)\right\} \geqq 0, \quad z \in U \text {. }
$$

If $f \in \Sigma$, it is known [4] that $f$ is univalent and $f(U)$ is a domain convex in the v-direction, i.e., the intersection of $f(U)$ with each vertical line is connected (or empty). Moreover, $f$ possesses the normalization

$$
\begin{aligned}
& \underset{z \rightarrow 1}{\limsup \operatorname{Re} f(z)}=\sup _{|z|<1} \operatorname{Re} f(z), \\
& \liminf _{z \rightarrow-1} \operatorname{Re} f(z)=\inf _{|z|<1} \operatorname{Re} f(z),
\end{aligned}
$$

meaning that the prime ends corresponding to $z= \pm 1$ are, in some sense, the right and left extremes of $f(U)$. In fact, the univalence, convexity in the $v$-direction, and normalization (2) characterize the class $\Sigma[4$, Theorem 1$]$.

We now determine the radius of convexity for the class $\Sigma$.

THEOREM 1. If $f \in \Sigma$, then $f$ maps $\{|z|<r\}$ onto a convex domain for $r \leqq c=\frac{1}{2}(1+\sqrt{ } 5)-\left[\frac{1}{2}(1+\sqrt{ } 5)\right]^{1 / 2}=.346 \cdots$. The constant $c$ is sharp for the function

Received by the editors July 13,1970 .

AMS 1970 subject classifications. Primary 30A32; Secondary 30A34.

Key words and phrases. Close-to-convex functions, convexity in one direction, radius of convexity, coefficient estimates, distortion theorems.

1 This research was supported in part by the National Research Council of Canada grant A-7339 and National Science Foundation grant GP-11603. 


$$
f(z)=\frac{i}{2} \log \frac{(1-i z)^{2}}{\left(1-z^{2}\right)},
$$

which is in $\mathbf{\Sigma}$.

Proof. Let $f \in \Sigma$ and define

$$
G(z)=\frac{\left(1-z^{2}\right) f^{\prime}(z)-1}{\left(1-z^{2}\right) f^{\prime}(z)+1} .
$$

Since $\operatorname{Re}\left\{\left(1-z^{2}\right) f^{\prime}(z)\right\} \geqq 0$, the function $G$ is analytic in $U$ and $|G| \leqq 1$. It is a consequence of Schwarz's lemma that

$$
\frac{\left|G^{\prime}(z)\right|}{1-|G(z)|^{2}} \leqq \frac{1}{1-|z|^{2}} .
$$

Hence, for $|z| \leqq r$,

$$
\begin{aligned}
\operatorname{Re}\left\{1+\frac{z f^{\prime \prime}(z)}{f^{\prime}(z)}\right\} & =\operatorname{Re}\left\{\frac{1+z^{2}}{1-z^{2}}+\frac{2 G z^{\prime}(z)}{1-G^{2}(z)}\right\} \\
& \geqq \frac{1-r^{2}}{1+r^{2}}-\frac{2 r}{1-r^{2}}=\frac{1-2 r-2 r^{2}-2 r^{3}+r^{4}}{1-r^{4}} .
\end{aligned}
$$

Using elementary calculus one verifies that $1-2 r-2 r^{2}-2 r^{3}+r^{4}$ is positive for $0 \leqq r<c=\frac{1}{2}(1+\sqrt{ } 5)-\left[\frac{1}{2}(1+\sqrt{ } 5)\right]^{1 / 2}=.346 \cdots$. Therefore $f(|z|<r)$ is convex for $r \leqq c$. The constant $c$ is best possible since $\operatorname{Re}\left\{1+z f^{\prime \prime}(z) / f^{\prime}(z)\right\}<0$ for $z=i r$ and $r \in(c, 1)$ for the function (3).

The study of mappings $f$ onto domains convex in the $v$-direction was introduced by Fejér [2] and developed extensively by Robertson in [7] and elsewhere, especially for the case where all the coefficients of $f$ are real. In the latter case the domains are symmetric with respect to the real axis and either $f$ or $-f$ belongs to $\Sigma$.

It should be noted that every domain $D$ convex in the $v$-direction does not necessarily admit a mapping in $\Sigma$. In particular, the normalization (2) requires the complement of $D$ to contain at least two vertical half-lines unbounded in opposite directions.

2. The class of close-to- $\Sigma$ functions. In [5] W. Kaplan introduced the class of close-to-convex functions, i.e., functions $F$ analytic in $U$ such that $\operatorname{Re}\left\{F^{\prime} / \varphi^{\prime}\right\}>0$ for some convex mapping $\varphi$. We make an analogous definition.

Definition. $A$ function $F$ analytic in $U$ is close-to- $\Sigma$ if there exists a mapping $f \in \Sigma$ such that 


$$
\operatorname{Re} \frac{F^{\prime}(z)}{f^{\prime}(z)} \geqq 0, \quad z \in U .
$$

If (4) vanishes at some point, then by the minimum principle for harmonic functions it vanishes identically. In that case either $i F \in \Sigma$, $-i F \in \Sigma$, or $F \equiv$ constant. If, on the other hand,

$$
\operatorname{Re} \frac{F^{\prime}(z)}{f^{\prime}(z)}>0, \quad z \in U,
$$

then

$$
\frac{\partial}{\partial v} \operatorname{Im} F \circ f^{-1}(w)=\operatorname{Re} \frac{F^{\prime}\left(f^{-1}(w)\right)}{f^{\prime}\left(f^{-1}(w)\right)}>0
$$

and hence the composition $F \circ f^{-1}$ maps each vertical line segment in $f(U)$, which is convex in the $v$-direction, onto an analytic arc that may be parametrized as a function $u=u(v)$.

If $F$ is close-to- $\Sigma$, then $F$ need not be univalent; however, $F$ is locally univalent (or constant). Theorem 1 gives rise to an estimate for the radius of univalence:

THEOREM 2. If $F$ is close-to- $\Sigma$ and nonconstant, then $F$ is univalent on the disk $\{|z|<c\}$ where $c$ is defined in Theorem 1 .

Proof. If $\operatorname{Re}\left\{F^{\prime} / f^{\prime}\right\}>0, f \in \Sigma$, then $g=F \circ f^{-1}$ is analytic on the convex set $f(|z|<c)$ and $\operatorname{Re} g^{\prime}>0$. This is the familiar criteria of Wolff-Noshiro-Warschawski [9], [6], [8] for univalence of $g$, from which univalence of $F=g \circ f$ on $\{|z|<c\}$ follows. If $\operatorname{Re}\left\{F^{\prime} / f^{\prime}\right\}$ vanishes at some point, then $F$ is univalent on $U$ since either $i F \in \Sigma$ or $-i F \in \Sigma$.

REMARK. It is interesting to note that the same lower bound $c$ was obtained by $\mathrm{W}$. Chase [1] for the radius of univalence of " 0 -close-tostar" functions.

3. Coefficient estimates. We note as a lemma some results we will use from [4].

LEMmA 1. If $f(z)=\sum_{n=0}^{\infty} a_{n} z^{n} \in \Sigma$, then

$$
\left|a_{n}\right| \leqq\left|a_{1}\right| \text { for } n \geqq 1
$$

and

$$
\frac{(1-r)\left|f^{\prime}(0)\right|}{(1+r)\left(1+r^{2}\right)} \leqq\left|f^{\prime}(z)\right| \leqq \frac{\left|f^{\prime}(0)\right|}{(1-r)^{2}} \text { for }|z| \leqq r<1
$$


Both (6) and the upper bound in (7) are sharp for the convex mapping $f(z)=z(1-z)^{-1}$, and the lower bound in (7) is sharp for the mapping (3).

THEOREM 3. If $F(z)=\sum_{n=0}^{\infty} A_{n} z^{n}$ is close-to- $\Sigma$, then

$$
\left|A_{n}\right| \leqq n\left|A_{1}\right| \text { for } n \geqq 1 \text {. }
$$

REMARK. The estimate (8) is sharp since the Koebe function $k(z)=z(1-z)^{-2}$ is close-to- $\Sigma$ relative to the function $f(z)=z(1-z)^{-1}$ in $\Sigma$.

Proof of Theorem 3. Suppose $\operatorname{Re}\left\{F^{\prime} / f^{\prime}\right\} \geqq 0$ where $f \in \Sigma$. If $i F \in \Sigma$ or $-i F \in \Sigma$, then $\left|A_{n}\right| \leqq\left|A_{1}\right|$ follows directly from Lemma 1 . We may therefore assume that (5) holds and define

$$
\begin{aligned}
h(z) & =\frac{\left[F^{\prime}(z) / f^{\prime}(z)\right]-\operatorname{Im}\left\{F^{\prime}(0) / f^{\prime}(0)\right\}}{\operatorname{Re}\left\{F^{\prime}(0) / f^{\prime}(0)\right\}} \\
& =1+\sum_{j=1}^{\infty} c_{j} z^{j} .
\end{aligned}
$$

Since $h$ is analytic in $U$ and has positive real part, it is well known $\left[3\right.$, p. 199] that $\left|c_{j}\right| \leqq 2$ for all $j$. From (9) we find

$$
\sum_{n=1}^{\infty} n A_{n} z^{n-1}=\sum_{n=1}^{\infty} n a_{n} z^{n-1}\left[\frac{A_{1}}{a_{1}}+\left(\operatorname{Re} \frac{A_{1}}{a_{1}}\right) \sum_{j=1}^{\infty} c_{j} z^{j}\right]
$$

and by equating coefficients

$$
n A_{n}=n \frac{a_{n}}{a_{1}} A_{1}+\left(\operatorname{Re} \frac{A_{1}}{a_{1}}\right) \sum_{k=1}^{n-1} k a_{k} c_{n-k} .
$$

Using the bound $\left|a_{n}\right| \leqq\left|a_{1}\right|$ from Lemma 1 and $\left|c_{j}\right| \leqq 2$, we have

$$
n\left|A_{n}\right| \leqq n\left|A_{1}\right|+n(n-1)\left|a_{1}\right|\left(\operatorname{Re} \frac{A_{1}}{a_{1}}\right) \leqq n^{2}\left|A_{1}\right| \text {. }
$$

\section{A distortion theorem.}

THEOREM 4. If $F$ is close-to- $\Sigma$, then for $|z| \leqq r<1$,

$$
\frac{(1-r)^{2}\left|F^{\prime}(0)\right|}{(1+r)^{2}\left(1+r^{2}\right)} \leqq\left|F^{\prime}(z)\right| \leqq \frac{(1+r)\left|F^{\prime}(0)\right|}{(1-r)^{3}}
$$

and

$$
|F(z)-F(0)| \leqq \frac{r\left|F^{\prime}(0)\right|}{(1-r)^{2}}
$$


and, for $|z|=r<c$,

$$
\left[\frac{2 r}{1+r}-\arctan r\right]\left|F^{\prime}(0)\right| \leqq|F(z)-F(0)| \text {. }
$$

REMARKs. Although functions which are close-to- $\Sigma$ need not be univalent, it is interesting that the upper bounds in (10) and (11) are the same as for the class of univalent functions. They are also sharp since the Koebe function is close-to- $\Sigma$. Moreover, the lower bounds in (10) and (12) are sharp for the function

$$
F(z)=\frac{2 z}{1-i z}+\frac{1}{2} \log \frac{1-z}{1+z},
$$

which is close-to- $\Sigma$ relative to the function (3).

As a consequence of (12) close-to- $\Sigma$ functions $F$ have the property that $F(U)$ contains the disk $\left\{|w|<\left|F^{\prime}(0)\right| d\right\}$ where $d=2 c(1+c)^{-1}$ $-\operatorname{arc} \tan c=.18 \cdots$.

Proof of Theorem 4. If $g$ is analytic in $U$ and $\operatorname{Re} g \geqq 0$, it follows from Schwarz's lemma (cf. [4, Lemma 5]) that $g$ has the representation

$$
g(z)=\frac{g(0)+\overline{g(0)} G(z)}{1-G(z)} \text { where }|G(z)| \leqq|z| .
$$

Suppose now that $g=F^{\prime} / f^{\prime}$ where $\operatorname{Re}\left\{F^{\prime} / f^{\prime}\right\} \geqq 0$ and $f \in \Sigma$. Then

$$
\left|F^{\prime}(z)\right|=\left|f^{\prime}(z)\right| \frac{F^{\prime}(0)}{f^{\prime}(0)}+\frac{\overline{F^{\prime}(0)}}{\overline{f^{\prime}(0)}} G(z)|| 1-\left.G(z)\right|^{-1} \text {. }
$$

Direct estimates of (15) together with (7) yield the bounds in (10). Inequalities (11) and (12) follow from (10) by standard integration arguments.

5. Concluding remarks. Theorems 2,3 , and 4 hold more generally for the class of functions $F$ analytic in $U$ and satisfying

$$
\operatorname{Re}\left\{e^{i \alpha} \frac{F^{\prime}}{f^{\prime}}\right\} \geqq 0
$$

for some $f \in \Sigma$ and $\alpha \in[0,2 \pi)$. Except for the degenerate case $\operatorname{Re}\left\{e^{i \alpha} F^{\prime} / f^{\prime}\right\} \equiv 0$, relation (16) means geometrically that components of horizontal lines in $e^{-i \alpha} f(U)$ are mapped by $F \circ\left(e^{-i \alpha} f\right)^{-1}$ onto analytic arcs that may be represented as functions $v=v(u)$ and, similarly, components of vertical lines are mapped onto arcs with representation $u=u(v)$. 


\section{REFERENCES}

1. W. Chase, p-close-to-star functions, Notices Amer. Math. Soc. 17 (1970), 172. Abstract \#672-315.

2. L. Fejér, Neue Eigenschaften der Mittelwerte bei den Fourierreihen, J. London Math. Soc. 8 (1933), 53-62.

3. G. M. Goluzin, Geometric theory of functions of a complex variable, GITTL, Moscow, 1952; English transl., Transl. Math. Monographs, vol. 26, Amer. Math. Soc., Providence, R.I., 1969. MR 15, 112.

4. W. Hengartner and G. Schober, On schlicht mappings to domains convex in one direction, Comment. Math. Helv. 45 (1970), 303-314.

5. W. Kaplan, Close-to-convex schlicht functions, Michigan Math. J. 1 (1952), 169185. MR 14, 966.

6. K. Noshiro, On the theory of schlicht functions, J. Fac. Sci. Hokkaido Univ. $2(1934 / 1935), 129-155$.

7. M. S. Robertson, On the theory of univalent functions, Ann. of Math. (2) 37 (1936), 374-408.

8. S. E. Warschawski, On the higher derivatives at the boundary in conformal mapping, Trans. Amer. Math. Soc. 38 (1935), 310-340.

9. J. Wolff, L'intégrale d'une fonction holomorphe et d partie réelle positive dans un demi-plan est univalente, C. R. Acad. Sci. Paris 198 (1934), 1209-1210.

Universite Laval, Quebec 10e, Qú́bec, Canada

INDIANA UnIVERSITy, BLOOMINGTON, Indiana 47401 